\title{
Características Epidemiológicas e Demográficas dos Doentes Portadores de Tumores da Cabeça e Pescoço no Norte de Portugal: Impacto na Sobrevivência
}

\author{
Epidemiological and Demographic Characteristics of \\ Patients with Head and Neck Tumours in the Northern \\ Portugal: Impact on Survival
}

\author{
Roberto ESTÊVÃO凶\1, Tiago SANTOS ${ }^{2}$, Ana FERREIRA ${ }^{3}$, Anabela MACHADO ${ }^{4}$, João FERNANDES ${ }^{4}$, \\ Eurico MONTEIRO 4 \\ Acta Med Port 2016 Oct;29(10):597-604 - http://dx.doi.org/10.20344/amp.7003
}

\section{RESUMO}

Introdução: Em Portugal não existem, ainda, estudos epidemiológicos e demográficos realizados em doentes portadores de tumores da cabeça e pescoço. Os objectivos desta análise são descrever os aspectos epidemiológicos e demográficos dos doentes portadores de tumores malignos da cabeça e do pescoço, referenciados ao serviço de Otorrinolaringologia de um centro oncológico do norte de Portugal e avaliar o impacto destas características na sobrevivência.

Material e Métodos: Estudo retrospectivo descritivo, dos doentes referenciados entre janeiro de 2011 e dezembro de 2013 ao Serviço de Otorrinolaringologia do Instituto Português de Oncologia do Porto. Foram incluídos 566 doentes. As variáveis analisadas foram a localização anatómica dos tumores, o estadiamento, o género e a idade dos doentes, o distrito de proveniência, os hábitos etílicos e tabágicos, o nível educacional, a profissão, o estado civil e a estrutura familiar.

Resultados: Os 566 doentes estudados correspondem a 498 homens e 68 mulheres, com idade média de 58,1 $\pm 12,2$ anos. A maioria $(80,5 \%)$ foi referenciada com doença em estadio avançado (III e IV). Verificamos hábitos etílicos moderados a excessivos em $78 \%$ dos doentes e $69 \%$ eram fumadores. A grande maioria dos doentes (82,3\%) tinha apenas o ensino básico. Os doentes com tumores da hipofaringe e com hábitos etílicos excessivos foram referenciados em estadios mais avançados e apresentaram pior sobrevivência $(p<0,001)$. Os doentes casados foram referenciados em estadios mais precoces $(p=0,020)$ e os doentes sem apoio familiar apresentaram pior sobrevivência $(p=0,030)$.

Discussão: Os dados epidemiológicos encontrados estão de acordo com a literatura internacional. A elevada taxa de doentes referenciados em estádio avançado pode ser atribuída ao atraso na procura de cuidados médicos ou a factores inerentes ao sistema de referenciação. A vigilância e o suporte familiar têm um papel fundamental na referenciação precoce e sobrevivência destes doentes.

Conclusão: Este estudo caracteriza um perfil populacional que pode beneficiar de maior grau de vigilância, de campanhas de consciencialização e/ou rastreios. Algumas das variáveis estudadas podem influenciar significativamente o estadio dos tumores e a sobrevivência.

Palavras-chave: Factores de Risco; Factores Socioeconómicos; Neoplasias de Cabeça e Pescoço/epidemiologia; Portugal.

\section{ABSTRACT}

Introduction: In Portugal there are still no epidemiological and demographic studies conducted in patients with of the head and neck tumors. The objectives of this analysis are to describe the epidemiological and demographics of patients with malignant tumors of the head and neck referred to an Ear, Nose and Throat department of an oncology center in the North of Portugal and also assess the impact of these characteristics on survival.

Material and Methods: Retrospective study of patients referred between January 2011 and December 2013 to the Ear, Nose and Throat Department of an oncology center (Instituto Português de Oncologia do Porto),. Five hundred sixty six patients were included. The analyzed variables were the anatomical location of the tumor, staging, gender and age of patients, the district of origin, the alcohol and tobacco consumption, educational level, occupation, marital status and family structure.

Results: The 566 patients studied (498 men and 68 women) presented a mean age of $58.1 \pm 12.2$ years. The majority (80.5\%) were referenced in advanced stage of disease (III and IV). Seventy eight per cent of patients had moderate or excessive alcohol consumption and $69 \%$ were smokers. The vast majority of patients $(82.3 \%)$ had only basic education. Patients with tumors of the hypopharynx and excessive drinking habits were referenced in more advanced stages and had a poorer survival $(p<0.001)$. Married patients were referred at earlier stages $(p=0.020)$ and patients without family support had poorer survival $(p=0.030)$.

Discussion: Epidemiological data are in agreement with the international literature. The high rate of patients referred with advanced stage of disease can be attributed to the patient's delay in seeking medical care or by factors inherent to the referral system. Surveillance and family support play a key role in early referral and survival of these patients.

Conclusion: This study features a population profile that can benefit from a higher degree of surveillance, awareness campaigns and/ or screenings. Some of the variables can significantly influence the tumor stage and survival.

Keywords: Head and Neck Neoplasms/epidemiology; Portugal; Risk Factors; Socioeconomic Factors.

1. Serviço de Otorrinolaringologia. Centro Hospitalar do Alto Ave. Guimarães. Portugal.

2. Serviço de Otorrinolaringologia. Centro Hospitalar Entre Douro e Vouga. Aveiro. Portugal.

3. Serviço de Assistência Social. Instituto Português de Oncologia do Porto. Porto. Portugal.

4. Serviço de Otorrinolaringologia. Instituto Português de Oncologia do Porto. Porto. Portugal.

$\square$ Autor correspondente: Roberto Estêvão. roberto_estevao@hotmail.com

Recebido: 14 de setembro de 2015 - Aceite: 16 de abril de 2016 | Copyright @ Ordem dos Médicos 2016 
INTRODUÇÃO

Os tumores da cabeça e do pescoço (TCP) agregam um grupo heterogéneo de neoplasias com origem nas mucosas de revestimento ou nas estruturas de suporte das vias aerodigestivas superiores. ${ }^{1}$ Englobam tumores localizados no lábio, na cavidade oral, na orofaringe, na hipofaringe, na laringe, no nariz e nos seios peri-nasais e nasofaringe, nas glândulas salivares, na tiróide e nas paratiróides. $O$ tipo histológico mais frequente é o carcinoma epidermóide que surge em mais de $90 \%$ dos casos. ${ }^{2}$

Estes tumores são o sexto tipo de cancro mais comum e um dos que apresenta pior taxa de sobrevivência. São diagnosticados anualmente à escala mundial cerca de 550 mil novos casos e este tipo histológico é responsável por cerca de 300 mil mortes por ano. ${ }^{3}$ Adicionalmente, a estes tumores são aplicadas modalidades terapêuticas associadas a elevada morbilidade, muitas vezes influenciando marcadamente o aspecto físico dos doentes e afectando funções básicas como a respiração, a deglutição e a fonação. ${ }^{1,4}$

O consumo de álcool e de tabaco, isoladamente ou em associação, são fatores etiológicos comuns para o aparecimento desses tumores quando localizados na cavidade oral, na orofaringe, na hipofaringe e na laringe, sendo por esta razão os mais importantes fatores de risco. ${ }^{5} \mathrm{~A}$ infecção pelo vírus humano do papiloma é actualmente considerada um importante fator etiológico para estas neoplasias, especialmente quando localizadas na orofaringe, e quando presentes em indivíduos mais jovens. ${ }^{5,6}$ A exposição ao pó de madeira, aos produtos utilizados na curtimenta de cabedais e ao níquel são fatores de risco conhecidos para o desenvolvimento de tumores no nariz e nos seios perinasais, ${ }^{7} \mathrm{e}$ a infecção pelo vírus Epstein-Barr está relacionada com os tumores localizados na nasofaringe. ${ }^{8}$

As características epidemiológicas dos doentes portadores de tumores da cabeça e do pescoço podem variar entre países e entre regiões de um mesmo país. Nos países desenvolvidos a incidência e a agressividade destes tumores tem vindo a diminuir ligeiramente enquanto nos subdesenvolvidos ou em vias de desenvolvimento a incidência e a mortalidade tendem a aumentar. ${ }^{9}$ Diversos estudos internacionais associam o aparecimento deste tipo de tumores com baixos estratos económicos e sociais. Uma meta análise recentemente publicada por um grupo de estudos multicêntrico estabelece que o baixo estrato sócio económico pode ser considerado como um fator de risco independente para determinados indivíduos poderem vir a contrair tumores nestas regiões. ${ }^{10} \mathrm{Em}$ Portugal não existem estudos específicos sobre este tema e, neste contexto, foi desenvolvido este estudo que tem como objetivo analisar os aspetos epidemiológicos e demográficos dos doentes portadores de tumores malignos da cabeça e do pescoço referenciados ao Serviço de Otorrinolaringologia (ORL) do Instituto Português de Oncologia (IPO) do Porto. Foi nossa intenção ainda avaliar a influência dessas características no estadio dos tumores e na sobrevivência global dos doentes, e ainda, alertar as instituições de saúde para um perfil demográfico e social destas populações, sobre as quais poderão ser implementados programas de prevenção e de rastreio.

\section{MATERIAL E MÉTODOS}

Após aprovação deste estudo pela Comissão de Ética da instituição, foi realizado um estudo retrospectivo descritivo procedendo-se à análise dos processos clínicos dos doentes referenciados ao Serviço de Otorrinolaringologia do IPO do Porto, entre janeiro de 2011 e dezembro de 2013.

Foram incluídos 566 doentes com o diagnóstico de carcinoma epidermóide da cavidade oral, da orofaringe, da laringe, da hipofaringe, do nariz e seios perinasais e da nasofaringe. Foram excluídos 24 doentes, 10 portadores de tumores benignos e de tumores malignos das glândulas salivares major, da tiróide e das paratiróides e 14 por dados insuficientes.

Os tumores foram estadiados segundo critérios clínicos e imagiológicos, de acordo com as directrizes da sétima edição do American Joint Committee on Cancer.

Os dados epidemiológicos avaliados foram o género, a idade, o distrito de proveniência e os hábitos etílicos e tabágicos. Os indicadores sociais analisados foram o nível educacional, a profissão, o estado civil e a estrutura familiar. Estas características foram avaliadas por uma assistente social.

Os dados foram recolhidos para uma folha de Microsoft Excel e, a análise estatística foi efectuada através do programa Statistical Package for the Social Sciences versão 20.0. Para a análise inferencial foi utilizado o teste de Qui quadrado e o método Kaplan-Meier complementado com o teste de log-rank. A medida de sobrevivência utilizada foi a sobrevivência global. Os resultados foram considerados estatisticamente significativos quando $p<0,05$.

Procedeu-se também a uma revisão da literatura publicada sobre o tema na base de dados da Medline para melhor enquadramento.

\section{RESULTADOS}

Foram estudados 566 doentes, correspondendo a 498 homens $(87,9 \%)$ e 68 mulheres, com idade média de 58,1 \pm 12,2 anos. Obtivemos a caracterização social de 308 doentes. O período médio de seguimento dos doentes desde a primeira à última consulta na instituição foi de $32,6 \pm 13,2$ meses.

Relativamente à localização dos tumores, a maioria $(56,5 \%)$ situava-se na orofaringe e na laringe, seguindo-se os tumores localizados na hipofaringe e na cavidade oral (Fig. 1).

No que diz respeito ao estadiamento, verificamos que os casos analisados se encontravam em estadios avançados de doença (80,5\% no estadio III e IV) (Fig.2).

Quanto à proveniência dos doentes, a maioria $(47,1 \%)$ foram referenciados do distrito do Porto, seguidos do distrito de Vila Real $(12,9 \%)$, Braga $(11,7 \%)$, Bragança $(11,4 \%)$, 
Viana do Castelo $(10,1 \%)$ e Aveiro (3,6\%). Os restantes $(3,2 \%)$ foram oriundos de outros distritos/regiões como Açores, Viseu e Guarda (Fig. 3).

Quanto aos hábitos, analisámos os padrões de consumo etílico e tabágico. Verificámos que a maioria $(91,2 \%)$ consumia álcool regularmente. Destes, 45,1\% consumia álcool em excesso (> $40 \mathrm{~g} / \mathrm{dia}$ ), 33,0\% apresentava padrões de consumo moderado (20 - $40 \mathrm{~g} / \mathrm{dia}), 13,1 \%$ ligeiro $(<20$ g/dia) e apenas 8,8\% não consumia bebidas alcoólicas.

Relativamente ao consumo de tabaco, $70,0 \%$ dos doentes eram fumadores e $11,0 \%$ ex-fumadores.

No que diz respeito ao estado civil a maioria dos doentes $(62,2 \%)$ eram casados, os restantes eram $17,7 \%$ solteiros, $11,3 \%$ divorciados, $8,8 \%$ viúvos. No entanto, analisando a estrutura familiar destes doentes constatamos que uma percentagem significativa dos doentes $(17,5 \%)$ fazia

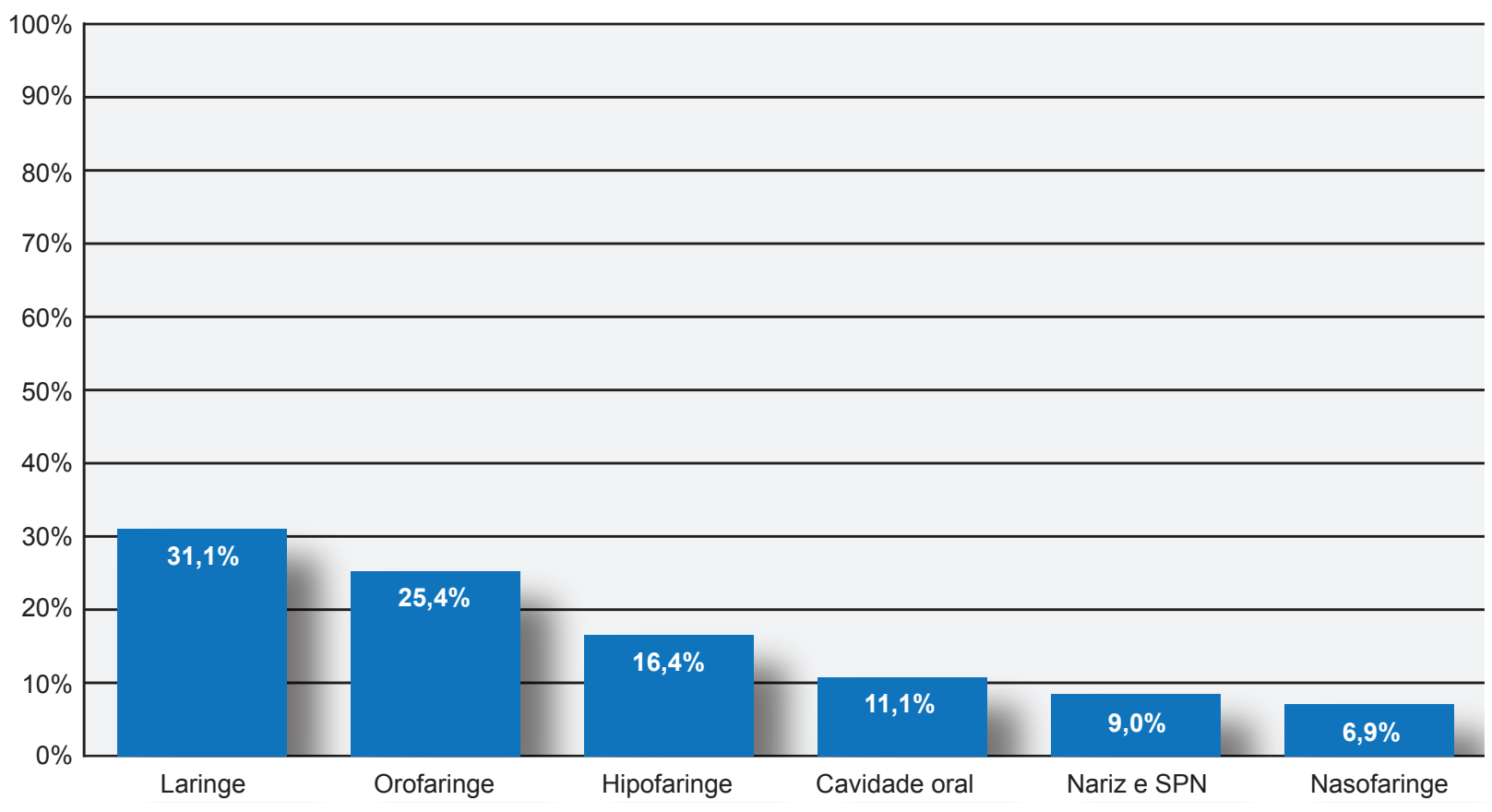

Figura 1 - Localização dos tumores

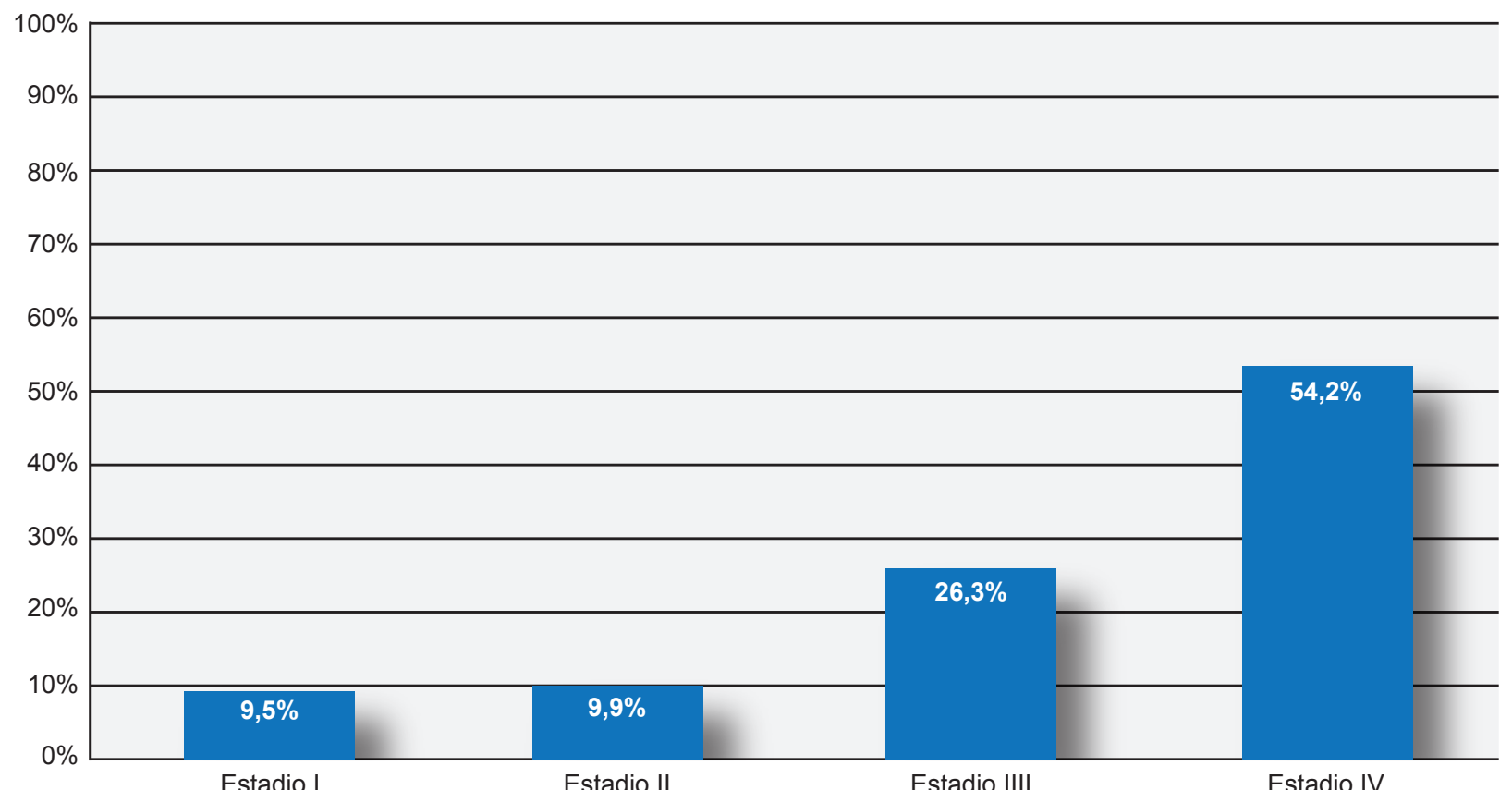

Figura 2 - Estadiamento tumoral 


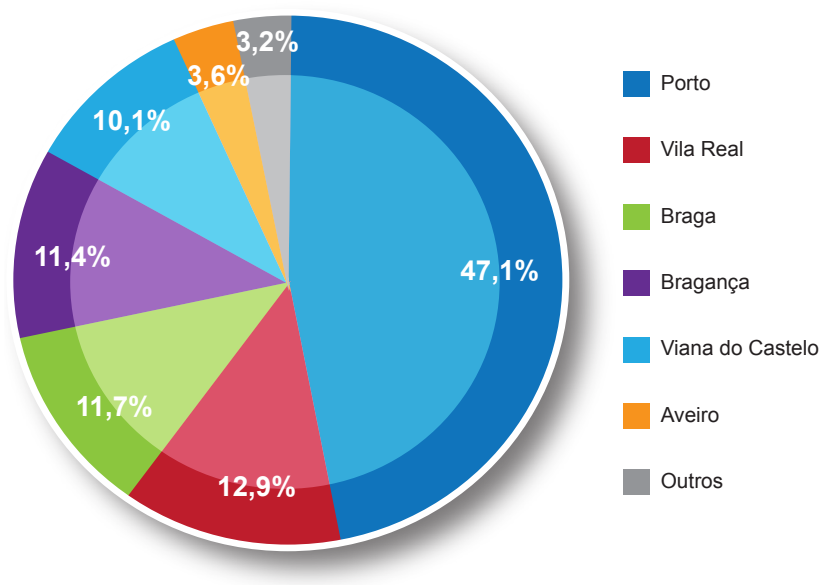

Figura 3 - Distrito de proveniência

parte de uma família unitária ou de cohabitação, referindo não terem apoio emocional ou económico por parte dos familiares.

Relativamente à escolaridade $(82,3 \%)$ tinha apenas o ensino básico e 6,7\% eram analfabetos. A maioria desempenhava profissões pouco diferenciadas (Fig. 4), sendo a construção civil a profissão mais frequentemente encontrada $(34,1 \%)$, seguida de operários fabris $(20,5 \%)$. Estes operários pertenciam a todo tipo de actividades, desde a indústria têxtil, do calçado, do curtume, à indústria madeireira e metalúrgica. Actividades relacionadas com a agricultura e pescas foram desempenhadas por $17,2 \%$ dos doentes, $10,1 \%$ desempenhavam profissões técnicas, $8,1 \%$ eram comerciantes e $10,1 \%$ desempenhavam outras profissões não incluídas nas categorias anteriores.

Verificamos, contudo, que apenas $30,8 \%$ dos doentes referenciados se encontravam profissionalmente activos.
Do grupo dos inativos, a maioria $(47,8 \%)$ estava reformado, $40,9 \%$ dependia de subsídio de doença/invalidez, e 11,3\% encontrava-se desempregado.

Correlacionando as diversas características epidemiológicas e sociais previamente descritas com o estadio e com a sobrevivência (Tabela 1) verificamos que os doentes com tumores da hipofaringe e com hábitos etílicos excessivos foram referenciados mais frequentemente em estadios avançados $(p<0,001)$. Em contrapartida os doentes casados foram referenciados em estadios mais precoces $(p=$ 0,020 ). A correlação entre as restantes características epidemiológicas e sociais e o estadio não obteve significância estatística.

No que diz respeito à associação entre as diversas características epidemiológicas e sociais e a sobrevivência, os factores que mais significativamente influenciaram a sobrevivência foram a localização do tumor $(p<0,001)$, a estrutura familiar $(p=0,030)$ e os hábitos etílicos $(p<$ 0,001 ). Doentes com tumores localizados $n$ cavidade oral e orofaringe e, na hipofaringe, os portadores de hábitos etílicos excessivos e os doentes sem apoio familiar foram os que apresentaram piores indicadores de sobrevivência e consequentemente pior prognóstico (Tabela 1; Fig. 5). De referir também que os doentes com hábitos tabágicos apresentaram tendencialmente pior prognóstico, embora sem significância estatística.

A sobrevivência aos 36 meses os diversos tipos de tumores por região anatómica foi de $58,9 \%$ para o cancro da hipofaringe, $68,0 \%$ para o cancro da cavidade oral, $74,8 \%$ para o cancro da orofaringe, $84,3 \%$ para o cancro da laringe, $87,6 \%$ para o cancro da nasofaringe e $91,5 \%$ para o cancro do nariz e seios perinasais.
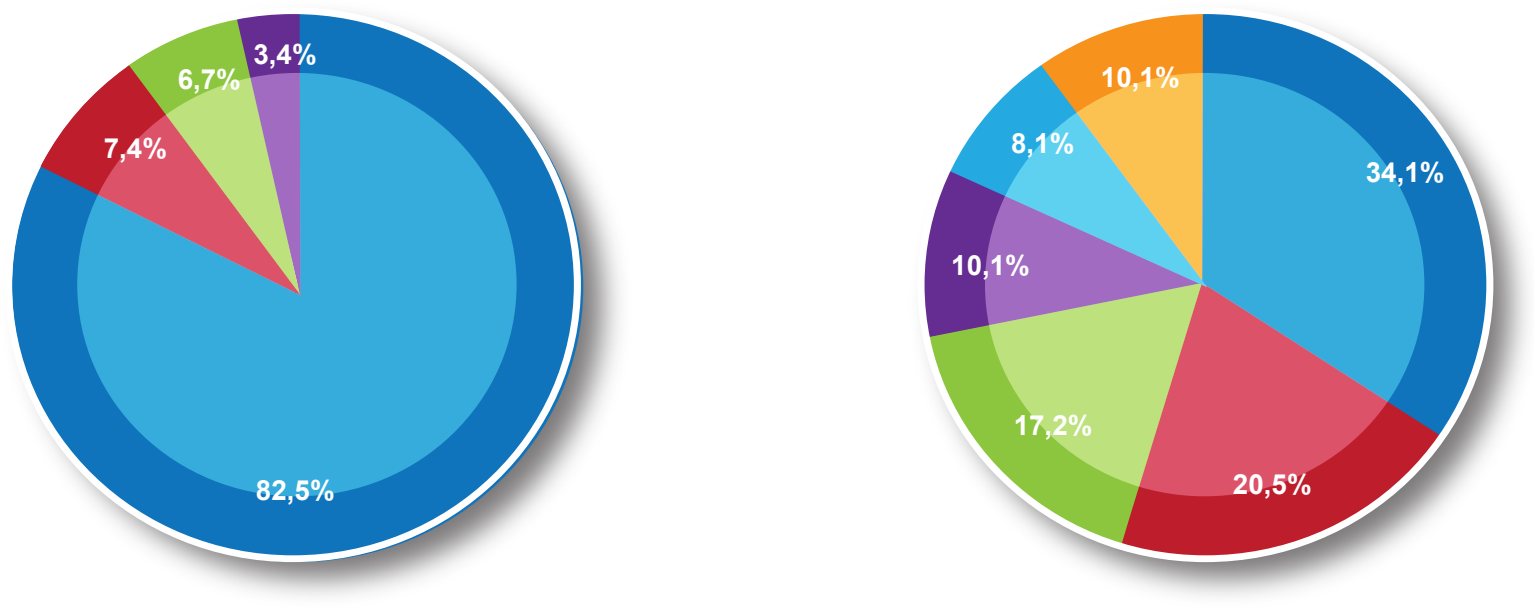

Ensino básico

Analfabetismo
Ensino secundário

Ensino universitário
Construção civil

Agricultura e pescas

Comerciantes
Operários fabris

Técnicos

Figura 4 - Nível educacional e profissão 
Tabela1 - Correlação entre as características epidemiológicas e sociais o estadio e a sobrevivência global

\begin{tabular}{|c|c|c|}
\hline Variáveis epidemiológicas e sociais & Correlação com o estadio tumoral & Correlação com a sobrevida \\
\hline Género & NS $(p=0,06)$ & NS $(p=0,12)$ \\
\hline Idade & NS $(p=0,07)$ & NS $(p=0,08)$ \\
\hline Localização do tumor & $\begin{array}{l}\text { Tumores da hipofaringe referenciados em } \\
\text { estádios mais avançados }(p<0,001)\end{array}$ & $\begin{array}{l}\text { Tumores da hipofaringe com pior } \\
\text { sobrevivência global }(p<0,001)\end{array}$ \\
\hline Distrito proveniência & NS $(p=0,07)$ & NS $(p=0,19)$ \\
\hline Hábitos etílicos & $\begin{array}{l}\text { Doentes com hábitos etílicos excessivos } \\
\text { referenciados em estádios mais avançados } \\
(p<0,001)\end{array}$ & $\begin{array}{l}\text { Doentes com hábitos etílicos excessivos } \\
\text { com pior sobrevivência global }(p<0,001)\end{array}$ \\
\hline Hábitos tabágicos & $N S(p=0,08)$ & NS $(p=0,09)$ \\
\hline Estado civil & $\begin{array}{l}\text { Casados referenciados em estádios mais } \\
\text { precoces }(p=0,02)\end{array}$ & NS $(p=0,07)$ \\
\hline Estrutura familiar & NS $(p=0,07)$ & $\begin{array}{l}\text { Doentes sem apoio familiar com pior } \\
\text { sobrevivência global }(p=0,03)\end{array}$ \\
\hline Educação & NS $(p=0,45)$ & NS $(p=0,90)$ \\
\hline Profissão & $\mathrm{NS}(p=0,11)$ & $\mathrm{NS}(p=0,33)$ \\
\hline
\end{tabular}

NS: Não significativo

\section{DISCUSSÃO}

Analisando os resultados obtidos verificamos que em geral estes dados epidemiológicos estão de acordo com o descrito na literatura internacional (maior incidência no género masculino e na sexta década de vida). ${ }^{11} \mathrm{O}$ mesmo se verificou para os hábitos etílicos e tabágicos. ${ }^{5,12,13}$ Alguns estudos apontam um tendencial aumento de incidência destes tumores no género feminino essencialmente devido a um aumento do consumo de álcool e de tabaco por parte das mulheres, ${ }^{11,14}$ aspecto ainda não evidenciado nesta série. Outros estudos referem um aumento da incidência do cancro da orofaringe em idades mais jovens essencialmente tentado relacionar o aumento da incidência deste tipo de tumores com o vírus do papiloma humano. ${ }^{5,6}$

Tal como esperávamos a localização mais frequente dos tumores foi a laringe, seguido da orofaringe. Contudo, a percentagem de doentes com cancro da cavidade oral pode estar subestimada, devido ao fato de doentes com este diagnóstico serem também referenciados e tratados noutros hospitais e noutros serviços deste hospital.

A percentagem de doentes $(80,5 \%)$ referenciada em estadios avançados (III e IV) está mais próxima do que é descrito em países em vias de desenvolvimento $(85,4 \%),{ }^{15}$ do que em países desenvolvidos (55\%). ${ }^{16}$ No entanto, a percentagem de doentes encontrada em estadios avançados no IPO do Porto pode não traduzir a realidade destes tumores na população portuguesa, dado o Serviço de ORL ser um centro de referenciação altamente diferenciado, recebendo portanto doentes provenientes de outros Hospitais concentrando casos mais complexos e portanto em estadios mais avançados. Por outro lado, pode estar em causa um atraso no diagnóstico ou na referenciação. Este atraso pode estar relacionado com o tempo que o doente leva desde que se apercebe que existe um problema até procurar ajuda médica, e/ou com o tempo de referenciação entre Medicina Geral e Familiar/ hospital distrital; hospital distrital/ IPO. Estes atrasos em geral estão associados a maiores taxas de mortalidade. ${ }^{17}$ Formas possíveis de reduzir estes atrasos na referenciação são a consciencialização e ou a realização de acções de sensibilização ou rastreios em populações de risco, ou ainda através de uma agilização do processo de referenciação.

No que diz respeito à proveniência dos doentes referenciados o principal distrito foi o Porto, seguido de Vila Real, Braga e Bragança. No entanto, estes dados não nos permitem inferir relativamente à incidência deste tipo de cancro nesses distritos, já que este dado diz respeito apenas aos doentes que são referenciados ao Serviço de ORL do IPO do Porto e não a todos os doentes com este diagnóstico que, podem ter sido referenciados a outros centros ou hospitais.

Apesar de a maioria dos doentes $(62,2 \%)$ ter o estatuto social de casado, concluímos através da análise social que uma percentagem significativa $(17,5 \%)$ de doentes fazia parte de famílias unitárias ou de coabitação referindo não possuir suporte emocional ou económico por parte de familiares. Salientamos que este dado não foi previamente descrito em outros estudos.

A população estudada apresenta um perfil educacional semelhante a outros artigos internacionais geralmente descrito como baixo. Contudo existe alguma variabilidade de país para país. Na Índia estão descritos, nestes doentes, níveis de analfabetismo de $34,6 \% .{ }^{16}$ Nos Estados Unidos da América, Johnson et $a^{1}{ }^{9}$ reportam uma maior incidência em doentes com escolaridade inferior ao ensino secundário. No nosso estudo a maioria dos doentes $(82,5 \%)$ completou apenas o ensino básico e constatamos uma taxa de analfabetismo de $6,7 \%$. 


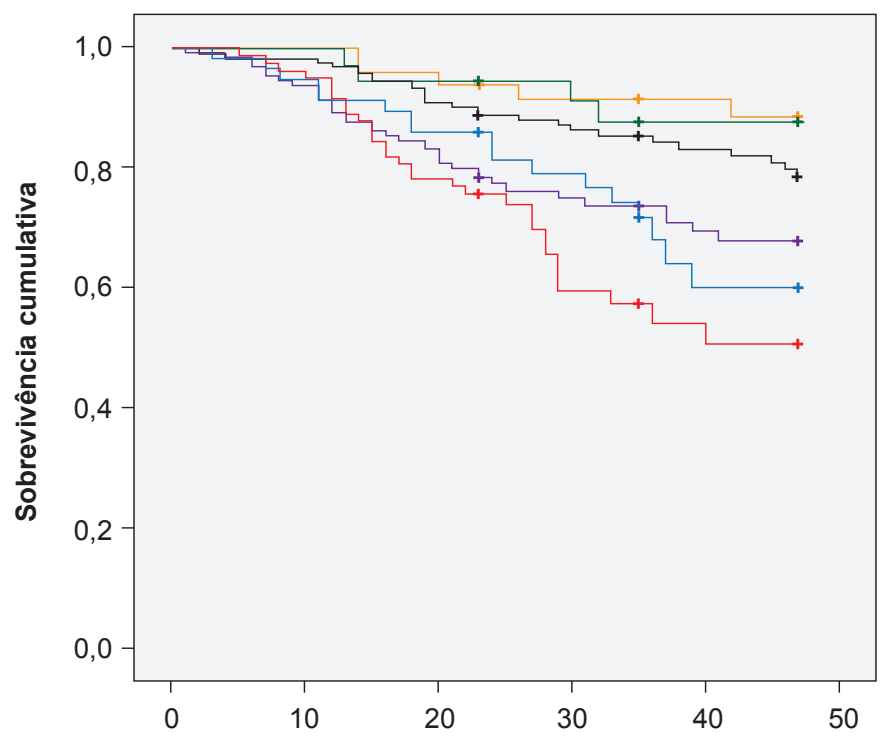

\section{Localização do tumor}

Nariz e seios perinasais

$\neg$ Nasofaringe

$\neg$ Laringe

$\neg$ Orofaringe

$\neg$ Cavidade oral

$\neg$ Hipofaringe

+ Nariz e seios perinasais - censored

+ Nasofaringe - censored

+ Laringe - censored

+ Orofaringe - censored

+ Cavidade oral - censored

+ Hipofaringe - censored

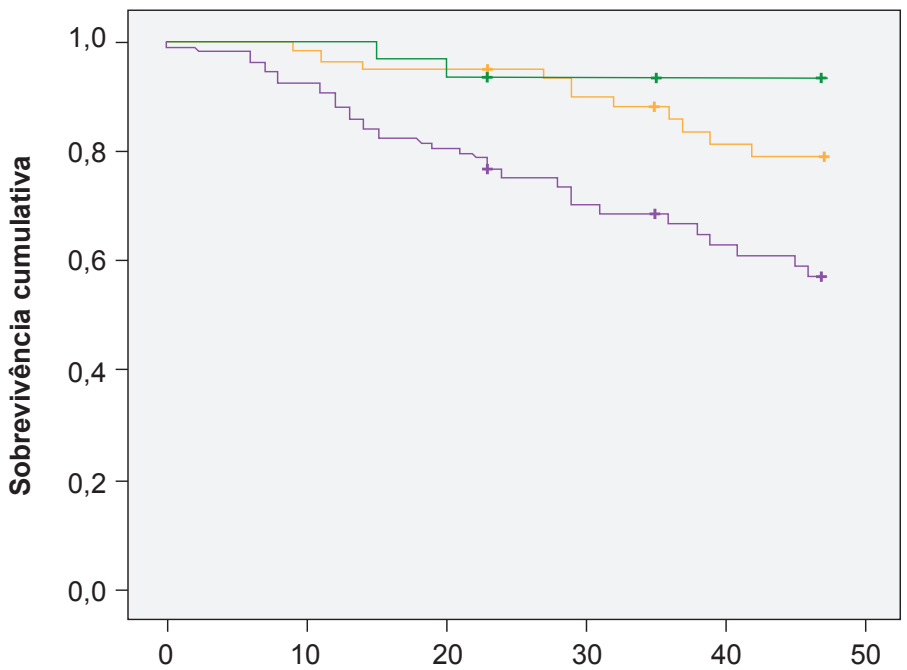

Hábitos etílicos

$\neg$ Ligeiros

Moderados

$\neg$ Excessivos

+ Ligeiros - censored

+ Moderados - censored

+ Excessivos - censored

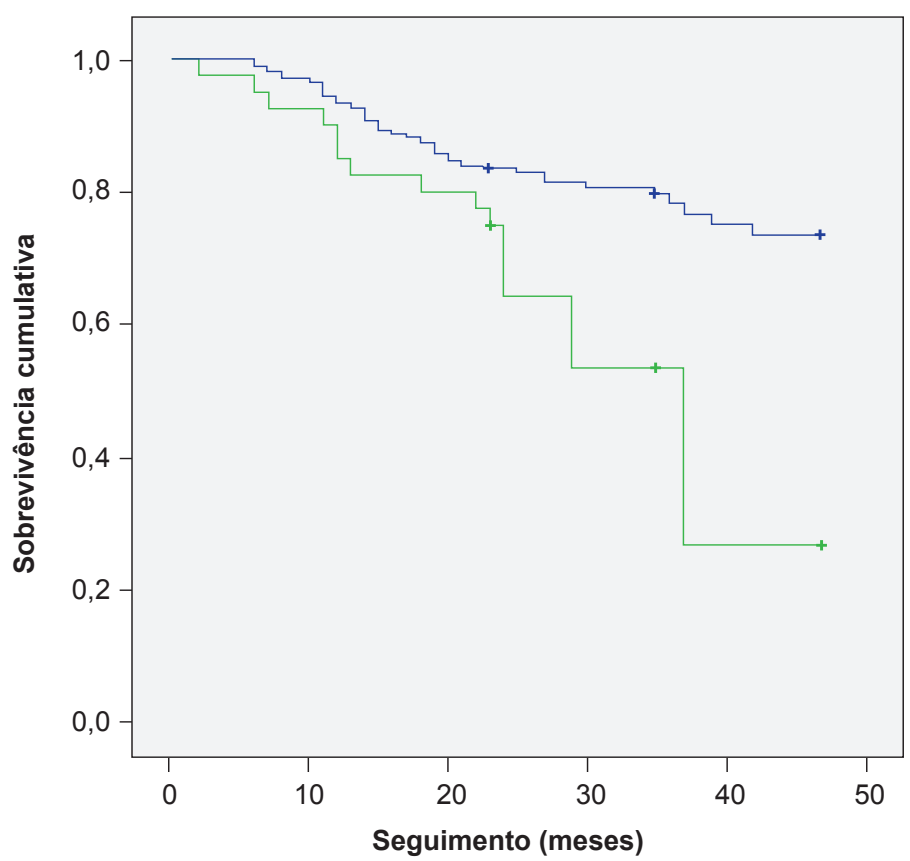

Estrutura famíliar

$\neg$ Com apoio familiar

$\neg$ Sem apoio familiar

+ Com apoio familiar - censored

+ Sem apoio familiar - censored

Figura 5 - Curvas de sobrevivência de acordo com a localização dos tumores, os hábitos etílicos e a estrutura familiar 
Relativamente à profissão, é de notar que a maioria dos doentes pertence a profissões pouco diferenciadas, com um grande incidência em trabalhadores da construção civil $(34,1 \%)$, operários fabris $(20,5 \%)$ e em agricultores e pescadores (17,2\%). Um estudo Europeu vem ao encontro destes resultados referindo também uma maior incidência destes tumores em trabalhadores da construção civil e em agricultores. ${ }^{18}$ Um outro estudo Francês também associa determinadas profissões como a agricultura e a indústria a um maior risco. ${ }^{19}$

No sentido de encontrar factores que pudessem influenciar o estadio dos tumores que nos foram referenciados fomos correlacionar os diversos factores epidemiológicos e sociais com o estadio. Obtivemos significância estatística relativamente à localização dos tumores, ao estado civil e aos hábitos etílicos dos doentes. Doentes com tumores da hipofaringe foram referenciados em estadios mais avançados. Estes tumores caracterizam-se por manifestarem sintomas tardiamente e por se apresentarem a tratamento quase sempre em estadios avançados. ${ }^{20}$ Quanto ao estado civil, verificamos neste estudo que os doentes casados se apresentaram em estadios mais precoces. De facto, diversos artigos vêm referenciando o eventual 'papel protector' do casamento nos carcinomas da cabeça e do pescoço, quer em outros tipos de tumores. ${ }^{21,22} \mathrm{O}$ facto de estes doentes serem referenciados em estádios mais precoces pode dever-se ao papel de vigilância do conjugue. ${ }^{22}$

Relativamente aos hábitos etílicos verificamos que os doentes com hábitos etílicos mais pronunciados foram referenciados mais frequentemente em estadios avançados. Brouha et $a^{13}$ chegaram a uma conclusão semelhante, referindo ainda que a mesma prática se vem observando em doentes com hábitos tabágicos excessivos. Na nossa análise a correlação não foi estatisticamente significativa embora tendencialmente os doentes com hábitos tabágicos excessivos também foram referenciados em estadios mais avançados.

No que diz respeito à correlação das várias características epidemiológicas e sociais com a sobrevivência global constatou-se que a localização dos tumores, os hábitos etílicos e a estrutura familiar influenciaram significativamente esta variável. Os doentes com tumores localizados na hipofaringe foram os que apresentaram pior sobrevivência e, os localizados no nariz, nos seios paranasais e na nasofaringe, os que apresentaram melhor prognóstico. De facto, o cancro da hipofaringe é considerada a forma tumoral com pior prognóstico, pois não se manifesta clinicamente em estadios iniciais, metastiza rapidamente e, apresenta frequentemente padrões de disseminação neural e submucosa. $^{20}$

Os doentes com hábitos etílicos excessivos (> $40 \mathrm{~g} / \mathrm{dia}$ ) também apresentaram piores indicadores de sobrevivên-

\section{REFERÊNCIAS}

1. Davies L, Welch GH. Epidemiology of head and neck cancer in the United States. Otolaryngol Head Neck Surg. 2006;135:451-7.

2. Galbiatti AL, Padovani-Junior JA, Maníglia JV, Rodrigues CD, Pavarino cia. Estes dados estão de acordo com um estudo realizado por Leoncini et al. ${ }^{23}$

Por último fomos analisar a influência da estrutura familiar na sobrevivência e verificamos que os doentes que referiam não possuir apoio familiar apresentaram piores índices de sobrevivência. Este dado não tem sido referido na literatura relativamente a este tipo de tumores e pode estar relacionado com a importância de um bom suporte familiar, aspecto que pode influenciar a adesão aos tratamentos, o cumprimento das medicações e, o controlo de outras comorbilidades.

\section{CONCLUSÃO}

Com esta análise salientamos o elevado número de doentes que são referenciados em estadio avançado de doença, o que tem implicações nos tratamentos e na sobrevivência. Descrevemos um perfil populacional composto essencialmente por doentes do sexo masculino na sexta década de vida, com hábitos etílicos e tabágicos acentuados, com baixo nível educacional, que desempenham profissões pouco diferenciadas e que podem beneficiar de maior vigilância, de campanhas de consciencialização ou de rastreios. Alertamos também para a necessidade de ser agilizado todo o sistema de referenciação destes casos.

Concluímos ainda que algumas variáveis epidemiológicas e sociais podem influenciar significativamente o estadio destes tumores ou até mesmo a sobrevivência.

\section{OBSERVAÇÕES}

Parte dos dados estatísticos deste estudo foram apresentados em comunicação livre, no dia 9 de junho de 2015, no $3^{\circ}$ Congresso Europeu de Otorrinolaringologia que decorreu em Praga, República Checa.

\section{PROTECÇÃO DE PESSOAS E ANIMAIS}

Os autores declaram que os procedimentos seguidos estavam de acordo com os regulamentos estabelecidos pelos responsáveis da Comissão de Investigação Clínica e Ética e de acordo com a Declaração de Helsínquia da Associação Médica Mundial.

\section{CONFIDENCIALIDADE DOS DADOS}

Os autores declaram ter seguido os protocolos do seu centro de trabalho acerca da publicação de dados.

\section{CONFLITOS DE INTERESSE}

Os autores declaram não terem qualquer conflito de interesse relativamente ao presente artigo.

\section{FONTES DE FINANCIAMENTO}

Os autores declaram não ter recebido subsídios ou bolsas para a elaboração do artigo.

ÉC, Goloni-Bertollo EM. Head and neck cancer: causes, prevention and treatment. Braz J Otorhinolaryngol. 2013;79:239-47.

3. Conway DI, Hashibe M, Boffetta P, Wunsch-Filho V, Muscat J, La 
Vecchia C, et al. INHANCE consortium. Enhacing epidemiologic research on head and neck cancer: INHANCE - The international head and neck cancer epidemiology Consortium. Oral Oncol. 2009;45:743-6.

4. Rinkel RN, Verdonck-de Leeuw IM, Doornaert P, Buter J, de Bree R, Langendijk JA, et al. Prevalence of swallowing and speech problems in daily life after chemoradiation for head and neck cancer based on cut-off scores of the patient-reported outcome measures SWAL-QOL and SHI. Eur Arch Otorhinolaryngol. 2016;273:1849-55.

5. Rettig EM, D'Souza G. Epidemiology of head and neck cancer. Surg Oncol Clin N Am. 2015;24:379-96.

6. Young D, Xiao CC, Murphy B, Moore M, Fakhry C, Day TA. Increase in head and neck cancer in younger patients due to human papillomavirus (HPV). Oral Oncol. 2015;51:727-30.

7. Binazzi A, Ferrante P, Marinaccio A. Occupational exposure and sinonasal cancer: a systematic review and meta-analysis. BMC Cancer. 2015;15:49.

8. Eduardo B, Raquel C, Rui M. Nasopharyngeal carcinoma in a south European population: epidemiological data and clinical aspects in Portugal. Eur Arch Otorhinolaryngol. 2010;267:1607-12.

9. Johnson S, McDonald JT, Corsten MJ. Socioeconomic factors in head and neck cancer. J Otolaryngol Head Neck Surg. 2008;37:597-601.

10. Conway DI, Brenner DR, McMahon AD, Macpherson LM, Agudo A, Ahrens W, et al. Estimating and explaining the effect of education and income on head and neck cancer risk: INHANCE consortium pooled analysis of 31 case-control studies from 27 countries. Int J Cancer. 2015;136:1125-39.

11. Ruback MJ, Galbiatti AL, Arantes LM, Marucci GH, Russo A, Ruiz-Cintra MT, et al.Clinical and epidemiological characteristics of patients in the head and neck surgery department of a university hospital. Sao Paulo Med J. 2012;130:307-13.

12. Potash AE, Karnell LH, Christensen AJ, Vander Weg MW, Funk GF. Continued alcohol use in patients with head and neck cancer. Head Neck. 2010;32:905-12.

13. Brouha X, Tromp D, Hordijk GJ, Winnubst J, De Leeuw R. Role of alcohol and smoking in diagnostic delay of head and neck cancer patients. Acta Otolaryngol. 2005;125:552-6.

14. La Vecchia C, Lucchini F, Negri E, Levi F. Trends in oral cancer mortality in Europe. Oral Oncol. 2004;40:433-9.

15. Krishnatreya M, Kataki AC, Sharma JD, Nandy P, Rahman T, Kumar $\mathrm{M}$, et al. Educational levels and delays in start of treatment for head and neck cancers in North-East India. Asian Pac J Cancer Prev. 2014;15:10867-9.

16. Dubois $M$, Ballivet de Régloix $S$, Raynal $M$, Lepage $P$, Kossowski M, Pons Y. Diagnosis and therapeutic pathways in head and neck cancers. Rev Laryngol Otol Rhinol. 2013;134:81-8.

17. Seoane J, Takkouche B, Varela-Centelles P, Tomás I, SeoaneRomero JM. Impact of delay in diagnosis on survival to head and neck carcinomas: a systematic review with meta-analysis. Clin Otolaryngol. 2012;37:99-106.

18. Richiardi L, Corbin M, Marron M, Ahrens W, Pohlabeln H, Lagiou P, et al. Occupation and risk of upper aerodigestive tract cancer: the ARCAGE study. Int J Cancer. 2012;130:2397-406.

19. Paget-Bailly S, Guida F, Carton M, Menvielle G, Radoï L, Cyr D, et al. Occupation and head and neck cancer risk in men: results from the ICARE study, a French population-based case-control study. J Occup Environ Med. 2013;55:1065-73.

20. Amar A, Curioni OA, Paiva DL, Rapoport A, Dedivitis RA, Cernea $\mathrm{CR}$, et al. Epidemiological assessment and therapeutic response in hypopharyngeal cancer. Braz J Otorhinolaryngol. 2013;79:500-4.

21. Aizer AA, Chen MH, McCarthy EP, Mendu ML, Koo S, Wilhite TJ, et al. Marital status and survival in patients with cancer. J Clin Oncol. 2013;31:3869-76.

22. Inverso G, Mahal BA, Aizer AA, Donoff RB, Chau NG, Haddad RI. Marital status and head and neck cancer outcomes. Cancer. 2015;121:1273-8.

23. Leoncini E, Vukovic V, Cadoni G, Pastorino R, Arzani D, Bosetti C, et al. Clinical features and prognostic factors in patients with head and neck cancer: Results from a multicentric study. Cancer Epidemiol. 2015;39:367-74. 